\title{
Analysis of TCPTranscription Factors Revealed Potential Roles in Plant Growth and Fusarium Oxysporum F.Sp. Cubense Tolerance in Banana (cv. Rasthali)
}

\section{Siddhant Chaturvedi}

National Agri-Food Biotechnology Institute

Shahirina Khan

National Agri-Food Biotechnology Institute

T. R. Usharani

Indian Institute of Horticultural Research

Siddharth Tiwari ( $\sim$ siddharth@nabi.res.in )

National Agri-Food Biotechnology Institute

\section{Research Article}

Keywords: Banana, Biotic stress, Fusarium oxysporum f. sp. cubense (Foc), Jasmonic acid, Phylogenetic analysis, TCP transcription factor.

Posted Date: September 15th, 2021

DOl: https://doi.org/10.21203/rs.3.rs-715375/v1

License: (c) (i) This work is licensed under a Creative Commons Attribution 4.0 International License.

Read Full License 


\section{Abstract}

The $T C P$ transcription factor gene family is highly conserved among the plant species. It plays a major role in the regulation of flower symmetry, cell division, development of leaf, fibre and nodule in the plants by controlling the synthesis of various plant hormones. Banana is a major staple crop in the world. However, Fusarium oxysporum f. sp. cubense (Foc) infection is a major threat to banana production. The role of $T C P$ gene family during the Foc infection is not explored till now. Herein, a total of 27 nonredundant TCP (MaTCP) gene sequences were retrieved from the banana genome and analysed for structural characteristics, phylogenetic correlation, subcellular, and chromosomal localizations. Phylogenetic analysis showed that the MaTCP proteins were highly conserved among different species and found to be the closest relative of the Oryza sativa and Zea mays. Promoter analysis of the TCP sequences showed that the cis-acting regulatory elements are associated with various stresses, environmental and hormonal signals. The higher transcripts accumulation in developing tissues (fruit finger, leaves, and stem) than of mature tissues (peel and pulp) showed a significant role of MaTCP in banana (cv. Rasthali) growth and development. Further, higher expression of the certain MaTCPS (MaTCP2, MaTCP4, MaTCP6, MaTCP9 and MaTCP11) in Foc race 1 infected root and leaf tissues of Rasthali indicated their promising role during Fusarium infection. This study will underpin the facet of TCP transcription factors on the development of biotic (Foc) stress tolerance in banana.

\section{Introduction}

The TEOSINTE BRANCHED/CYCLOIDEA/PROLIFERATING CELL FACTOR(TCP) transcription factors (TFs) encode the plant-specific proteins which serve a major role in plant growth and development by overseeing the modulation of plant hormones. They contain a conserved TCP domain which consists of 55-59 amino acids (aa) long basic helix loop helix (bHLH) motif $[1,2]$. The name TCP is derived from the Teosinate Branched1 (TB1) from the maize (Zea mays), Cycloidea ( $C Y C)$ from the Snapdragon (Antirrhinum majus), and Proliferating Cell Factors 1 and 2 (PCF 1 and 2) from the rice (Oryza sativa) [2]. On the basis of sequence homology, the TCPTF family is divided into two subfamilies viz., class I and class II. These can further be categorized into subclasses, such as class I into the PCF1 and PCF2 and class II in the CYC/TB1 and CINCINATA (CIN) $[1,3,4]$. The deletion of four aa in the basic region of class I and the presence of arginine-rich $\mathrm{R}$ domain and/or stretch of glutamic acid-cysteine-glutamic acid (ECE) in class II at 9, 10, 11 and 12th positions made the major differences in TCP proteins [2, 5]. TCPTFs are known to play both antagonistic and synergistic roles in plant growth and development $[2,6]$. For instance, the AtTCP2O of class I is reported to promote cell proliferation in the leaves, therefore, regulates plant growth and development [7]. A point mutation in the conserved region in the AtTCP20 sequence did not make any phenotypic changes in plants perhaps due to genetic redundancy [8]. However, the fusion of AtTCP20 protein with ethylene-responsive element binding factor-associated amphiphilic repression (EAR) motif inactivated the function of class I TFs thus, altered the phenotypes of plants [8]. TFs of class II are mostly involved in lateral organ development, floral transition and organ identity. Further, the class II TFs also play an inhibitory role in cell division, hence regulated plant development and promotes ageing 
[2]. The TB1 gene that is also known as the domestication gene in the maize inhibits the growth of lateral branches, while the loss of function of the $t b 1$ promotes lateral branches development [1]. The TB1 is also reported to be involved in the formation of female inflorescence in maize [9]. The $C Y C$ is considered another gene of class II and reported to regulate the asymmetry of petals and stamens in snapdragon, Gerbera and Linaria vulgaris [1]. The role of TCPTFs has also been demonstrated in leaf morphogenesis, seed germination, leaf branching, circadian rhythm, abiotic and biotic stresses $[2,10]$. The TCP can also interact with other TFs like DELLA, MYB and basic helix loop helix $(b H L H)$ that trigger the biosynthesis of auxin, flavonoid and maintain the apical meristem identity [11]. The TCPTFs can make a cross-talk and modulate the biosynthesis of plant hormones such as auxin, gibberellin, jasmonic acid, strigolactones, brassinosteroids and cytokinin [12-14].

Jasmonic acid (JA) is known to be involved in the various stresses and plant-microbe interaction mechanisms $[15,16]$. The JA biosynthesis pathway was reported to be hijacked at the time of the Fusarium attack in Arabidopsis and tomato plants. It suggests that the JA phytohormone is involved in wilt disease susceptibility $[17,18]$. The JA biosynthesis from alpha-linolenic acid is mediated through a chloroplast enzyme encoded by the lipoxygenase 2 (LOX2) gene. It was reported that AtTCP4 and AtTCP20 up and down-regulated expression of the LOX2 gene, respectively [19]. Therefore, modulation in the expression of the $L O X 2$ gene by $T C P$ can influence JA biosynthesis that subsequently affects plant response during Fusarium oxysporum f. sp. cubense (Foc) infection.

Banana is one of the most consumed fruit crops and the major backbone of the economy in several developing countries in the world. It is considered a staple food crop for millions of people and available throughout the year at a reasonable price in the market. In 2018, the total international market for bananas was US\$8 billion [20]. However, Foc race 1 causes serious damage to banana production and creates widely known Panama disease. Another devastating strain Foc tropical race 4 (Foc TR4) is reported in Australia and the Indian subcontinent, for which most of the cultivated banana crops are found to be susceptible [21]. It was reported that Foc race 1 alone causes the loss of more than USD 2.3 billion and wiped out the Gros Michel banana from the world export market. The outbreak of Foc TR4 affected more than 4,40,000 hectares (ha) of banana plantations around the world with the loss of more than USD 388 million [22]. It had estimated that in the wake of FOC TR4 the banana production would face a reduction of 2.8 million tonnes and that will increase the banana price up to 9.2 percent [22].

Rasthali (AAB group, Silk banana) is an Indian banana cultivar (cv.) with good flavour and quality but known to be highly susceptible to Fusarium race 1 [23]. In the present study, a comprehensive in-silico analysis of the TCP (MaTCP) TF gene family was performed in banana. The expression analysis of the TCPTFs in different tissues, and during the Foc race 1 infection in the leaf and root tissues of $\mathrm{cv}$.

Rasthali indicated their promising role in developmental biology and biotic stress management. These results can implicit a framework for further functional characterization of selected MaTCPTFs, in particular against the fusarium infection.

\section{Methods}




\section{Identification and sequence alignment of putative MaTCP from Musa acuminata}

The reported TCP sequences of Arabidopsisthaliana (24) and Oryza sativa (21) were retrieved from Arabidopsis database TAIR (http://www.arabidopsis.org) and rice genome annotation project (http://rice.plantbiology.msu.edu/index.shtml), respectively. Retrieved protein sequences were used as a query in blastp against the banana (Musaacuminata) proteome database [24] (http://bananagenome.cirad.fr/). The hidden markov model profiles of the TCP domain (PF03634) obtained from pfam (http://pfam.xfam.org) [25] was also used as a query to identify TCP proteins in Musa acuminata genome. The protein sequences were further checked with the conserved domain database (CDD) using the National Center for Biotechnology Information (NCBI) BLAST program and Simple Modular Architecture Research Tool (SMART) (http://smart.embl-heidelberg.de/). Additionally, physicochemical parameters like theoretical isoelectric point ( $\mathrm{pl})$, the number of aa, instability index, molecular weight, subcellular localization were predicted by ProtParam (https://web.expasy.org/protparam/) [26]. Subcellular localization of MaTCP genes was predicted by CELLO 2.0 (http://cello.life.nctu.edu.tw/).

\section{Phylogenic and synteny analysis of MaTCP sequences}

Multiple sequence alignment of MaTCP proteins was performed using Clustal X 2.0 (http://www.clustal.org/clustal2/) [27]. Phylogenetic tree was constructed to attain more understanding about the evolutionary relationship among Musa acuminata and diversified monocot, dicot and gymnosperm plant species. Protein sequence of dicots (Arabidopsis thaliana, Arachis hypogea, Beta vulgaris, Nicotiana tobaccum, Utricularia gibba), monocots (Brachypodium distachyom, Hordeum vulgare, Oryza sativa var. indica, Triticum aestivum, and Zea mays) and gymnosperm (Pinus taeda) were downloaded from the plant TF database (http://planttfdb.gao-lab.org/index.php). The tree was made using MEGA X software [28] with the neighbour-joining method using the pairwise deletion option, 1000 replicates of bootstrap and poisson correction distance parameters. An unrooted tree was constructed by using iTOL (Leutinic and Bork, 2007). Synteny analysis was done using the circos tool (http://circos.ca/) [29].

\section{Chromosomal location, gene structure and conserved motifs analysis}

The chromosome map of the banana genome was drawn using the information from NCBI. The MaTCP genes were mapped onto the corresponding chromosome using Map Gene2 Chromosome V2 (http://mg2c.iask.in/mg2c_v2.0/) [24]. The coding (CDS) and genomic sequences of the MaTCP were retrieved from the banana genome hub (http://banana-genome.cirad.fr/) (D'hont et al. 2012) for the exonintron configuration by using Genome Structure and Display Server (GSDS 2.0, http://gsds1.cbi.pku.edu.cn/) [30]. The analysis of the conserved motif of MaTCP proteins was done by using the MEME (http://meme-suite.org/) program [31].

\section{Cis-acting element analysis, synteny and gene ontology}


The cis-acting DNA elements analysis of promoter sequence (1500 bp upstream regions from coding sequence) were performed using the PlantCARE online program (https://sogo.dna.affrc.go.jp/). The functional annotation of identified TCP proteins was analyzed with blast2go tools [32]

\section{Plants infection with Foc race 1 and collection of tissue}

Banana cv. Rasthali is highly susceptible to the Foc race 1 infection [33]. The tissue culture raised 15-18 $\mathrm{cm}$ long Rasthali plantlets at 4-5 leaf stages were infected by dipping them in the Foc race 1 spore suspension that was colonized with millet grain (spore load $\sim 2 * 10^{8}$ ). After inoculation, plants were maintained in the greenhouse at $30-35^{\circ} \mathrm{C}$ at $70-75 \%$ relative humidity [34]. Root and leaf tissues were collected after 4 weeks of infected and uninfected control plants. For expression analysis in growing tissues viz., leaf, fruit finger, pseudostem, root, unripe peel, unripe pulp, ripe peel and ripe pulp were collected from the National Agri-Food Biotechnology Institute (NABI), Mohali research field-grown Rasthali plants. Without further ado, all tissue samples were quickly frozen in liquid nitrogen and kept at $-80^{\circ} \mathrm{C}$.

\section{RNA extraction, preparation of CDNA and qRT-PCR}

RNA from all the tissues was isolated by the plant total RNA extraction kit (Sigma-Aldrich, USA). DNase treatment was given to RNA by DNase I kit (Thermo Fisher Scientific, USA) to get rid of DNA contamination. RNA was checked and quantified for integrity and intactness on agarose gel and Nanoquant, respectively (Thermo Fisher Scientific, USA). DNase treated RNA was used to synthesize firststrand cDNA synthesis with revert aid first-strand cDNA synthesis kit (Thermo Fisher Scientific, USA). A CFX96 touch real-time PCR detection system (Bio-Rad, USA) was used to carry out quantitative real-time PCR (qRT-PCR) analysis. Normalization of different selected genes was done using actin1 (GenBank Accession No. AF246288) as a housekeeping gene [35]. The qRT-PCR reaction was conducted using a $2 X$ SYBR green master mix (Bio-Rad, USA). The comparative expression level was investigated by applying the $2^{-\Delta \Delta C t}$ method [36]. The primers list used in expression analysis is mentioned in the Table S1.

\section{Results And Discussion}

\section{Identification and sequence alignment of putative MaTCPTFs}

The TFs play important roles in various aspects of the biological and physiological processes of plant growth and development. TCPTFs gene family is involved in numerous prospects of plant growth and development. For instance, they help in fibre development in cotton [37], nodule formation in common bean [38], abiotic stress responses in maize [39] and Panicum [4]. TCPTFs have also reported to involved in ripening and abiotic stress (cold) tolerance in banana [40, 41], but did not enlighten their role in biotic stress and development processes. In the present study, a total of $45 \mathrm{MaTCP}$ sequences were identified in the banana genome database. To remove redundancy, we kept sequences that have shown at least $50 \%$ identity in the blast with Arabidopsis thaliana. A recursive blast search was further used to remove duplicate entries. Besides, sequences were further explored by considering the occurrence of the 
conserved TCP domain. Finally, a total of 27 MaTCP sequences were considered and designated as MaTCP1 to MaTCP27 according to their genomic position (Fig. S1). The number of MaTCPs was found in accordance with other crops like Vitis vinifera (18), Medicago (21), Oryza sativa (22) and Arabidopsis (24). While, Panicum (42), Nicotiana (61) and Gossypium barbedense (75) have a higher number of TCPs $[2,3]$. The number of TFs might be governed on the basis of genome complexity of the plants.

The physicochemical properties of MaTCP were encompassed in the Table S2. The base length of MaTCPCDS ranged between $744 \mathrm{bp}$ (MaTCP3) to $1821 \mathrm{bp}$ (MaTCP13). The predicted aa length varies from 248 (MaTCP3) to 607 (MaTCP13) with a mean length of 361 aa. The pl was in the range of 5.51 (MaTCP1) to 10.12 (MaTCP5) with a mean value of 7.80. The predicted molecular mass of proteins ranged from $25 \mathrm{kDa}$ (MaTCP3) to $65 \mathrm{kDa}$ (MaTCP13) with an average molecular mass of 38kDa. All the MaTCP sequences were found to be localized in the nucleus. Domain analysis of MaTCP proteins revealed the presence of TCP domain, which is composed of a highly conserved structure of bHLH motif and have 54 to 59 aa long residue (Fig. 1a).

The sequence alignments showed the presence of four conserved regions (one basic, two helix and one loop) in the bHLH domain of all MaTCP proteins (Fig. 1a). The sequence logo of the conserved region is shown in (Fig. 1b). All MaTCP proteins were divided into class I that has comprised of 16 members and class II having 11 members. The class II proteins have shown an additional 4 aa, which are absent in class I. Later, Class II proteins were split up into two subfamilies i.e., CYC/TB1 and CIN, based on the presence of unlike aa. Total 3 members of class II fall in the CYC/TB1 and 8 members came under the CIN group.

\section{Phylogenetic relationship and synteny analysis of MaTCP}

TCP proteins were characterized in Arabidopsis and rice [7, 19, 42]. However, the biological functions of most of the MaTCPs are not understood yet. A total of 322 TCP protein sequences from Arabidopsis thaliana, Arachis hypogea, Beta vulgaris, Nicotiana tobaccum, Utricularia gibba, Brachypodium distachyom, Hordeum vulgare, Oryza sativa var. indica, Triticum aestivum, and Zea mays and Pinus taeda were aligned with banana derived sequences for the construction of the phylogenetic tree (Fig. 2). The analysis showed that class I contained 139 members representing $43.16 \%$ of total TCP proteins and class II confined with 183 (56.84\%) members. The proteins of class II bifurcated into two subclasses i.e., CIN comprised of 124 (38.50\%) and CYC/TB1 with 59 (18\%). The distribution of MaTCP proteins among subfamilies is found to be uneven and showed that class I have 16 while class II contained only 11 MaTCP proteins (Fig. 2). In an evolutionary relationship, Oryza sativa and Zea mays derived sequences were showed close relation with all MaTCP due to the taxonomical similarity of these crops, while other species exhibited distant genetic relation with banana sequences. Furthermore, the comparative synteny maps between banana and Arabidopsis suggested that most of the banana TCPs have orthologs in Arabidopsis (Fig. 3).

\section{Chromosomal distribution, exon-intron configuration and conserved motif analysis}


All 27 MaTCPTFs were asymmetrically dispersed in the banana genome (Fig. S1). The distribution of MaTCP on the chromosome varies between 2 to 4 . Chromosomes 1, 2, 8 and 10 contain 2 genes, chromosomes 3, 5, 6, 7 and 11 located with 3 genes, chromosome 2 contain 4 genes, while no MaTCP was found on chromosome 9 .

Coding and genomic sequences of $M a T C P$ were used to predict the exon-intron pattern (Fig. 4). It revealed that 25 MaTCP were intron-less, except MaTCP13 and MaTCP18 that contain 2 and 1 intron, respectively. Together with cell envelope and aa biosynthesis, the character of intronless genes are an adjunct of translation and energy metabolism function [43]. Gene ontology of the intronless gene family showed that they are mainly involved in growth factor, immune response, transcription regulation and structural proteins [43]. Exon analysis showed that most of the genes contain the same length of exons which gave an idea that most of the genes evolved from the same ancestors. Similar observations have been reported in Gossypium barbadense [44], Nicotiana tabacum [3] and Vitis vinifera [45] plants. The diversity of motif compositions among MaTCP showed the presence of the six conserved motifs. The prevalence of the highly conserved TCP domain (motif 1 ) was depicted in all MaTCP proteins (Fig. 5). Class II members of MaTCP was characterized by the presence of motif 3 at $\mathrm{N}$ - terminal, while motif 2 was absent from class II members. Motif 5 was conserved only in MaTCP7, MaTCP13, MaTCP16 and MaTCP23 (Fig. S2) that belongs to class II members. Motif 1 was found to be conserved in all MaTCP members thus, it might be essential to perform the crucial functions. In crops like Gossypium barbadense [44], Vitis vinifera [45] have also shown conserved motif 1 in most of the TCP members. Motif 5 was exclusively present in the CIN family of class II. Hence, the specificity of motifs to certain groups indicated that the diverse function of TCP proteins might be dependent on the presence of explicit conserved motif.

\section{Cis-acting element analysis and gene ontology}

The analysis of the cis-regulatory elements showed the presence of CAAT and TATA boxes in all MaTCP promoters (Table S3). These boxes have a role in the transcriptional regulation of the gene [46]. Other cisregulatory elements for phytohormone, plant growth and development, and stress responses were also present in the promoters. The HDZip was found in the MaTCP11 promoter and reported to be involved in the differentiation of mesophyll palisade tissue [47]. Hormone-related cis-acting elements, such as methyl jasmonate responsive elements (CGTCA and TGACT element), abscisic acid response elements (ABRE), gibberellin responsive elements (P-box, GARE) and auxin-responsive element (TGA) were found exclusively in promoters region of MaTCP7, MaTCP12, MaTCP14, MaTCP15, MaTCP16, MaTCP17, MaTCP19, MaTCP23, MaTCP24, MaTCP25 and MaTCP26. Previously, a similar type of cis-regulatory element patterns was examined in grapevines [45] and switchgrass [4]. Therefore, the presence of these regulatory elements in $T C P$ promoters indicated their role in hormone synthesis and, the growth and development of plants. Further, the stress-related cis-acting elements i.e., drought inducibility (MBS) and low temperature (LTR) responsiveness element were specifically found in MaTCP7, MaTCP16, MaTCP19 and MaTCP15, MaTCP23, respectively. The presence of zein metabolism regulation element (02 site) in 
the promoter region of MaTCP23 TF has hinted its role in abiotic stress resistance, carbon and amino acid biosynthesis [48].

All 27 MaTCP TFs were annotated and categorized into three main gene ontology (GO) categories those are biological process, molecular function and cellular component (Fig. S3). MaTCP were mostly involved in the regulation of transcription (79\%), then in flavonoid biosynthesis (9\%), regulation of shoot $(6 \%)$ and secondary shoot formation (6\%). In molecular functions, most of the represented terms were transcriptional regulation (55\%), DNA binding activity (38\%) and catalytic activity (3\%). In terms of cellular component, proteins were distributed in the nucleus (93\%) and integral membrane (7\%).

\section{Expression analysis of MaTCPTFs under Foc race 1 treated tissues of banana}

Expression analysis was conducted in Foc race 1 infected leaf and root with compared to uninfected healthy (control) samples of the same tissues. The higher transcripts expression of MaTCP2 (4 fold), MaTCP6 (5.1 fold), MaTCP18 (1.52 fold), MaTCP21 (2.90 fold) and MaTCP24 (3.10 fold) was noted in the root tissue than of control (Fig. 6 and Fig. S4a). However, other TFs have shown similar expression patterns to control. Fusarium is a soil-borne fungus that reproduces through the asexual method by producing microconidia, macroconidia and chlamydospores [49]. Fusarium can live in the soil for a long duration in the dormant stage, hence it is hard to eradicate its infection from the soil [50]. Presumably, this might be the reason that the FoC attack in banana plants has increased the expression of certain TCP TFs in the root tissue. The higher expression of MaTCP1 (3.9 fold), MaTCP4 (5.02 fold), MaTCP9 (5.71 fold), MaTCP10 (1.5 fold), MaTCP11 (16 fold) and MaTCP19 (2.5 fold) found in the Foc race 1 infected leaf than in control (Fig. 6 and Fig. S4b). The higher expression of certain TCPTFs in leaf showed that they might have a broad role in growth and development, and stress management in banana plants. A similar observation was reported in the potato where expression of the StTCP23 was modulated during the infection of Streptomyces turgidiscabies (scad disease) and Fusarium solani. The susceptible and lethal symptoms of scad disease were significantly increased when StTCP23 was silenced in the potato [51]. It can be inferred from the present analysis in banana that after Foc infection in the root, plant tissues like leaf can sense the stress and triggered expression of the certain TCP (MaTCP4, MaTCP9 and MaTCP11). It is also intended to mitigate the biotic stress response by the higher expression of these TCP TFs in the infected banana plant. Fusarium management is one of the most important aspects of banana cultivation as its infection is lethal and once the plant got infected it spread rapidly and can damage the whole crop [50]. This soil-borne facultative fungus is responsible for deadly wilt disease in more than 100 crop plants. Hence, the present study was explored to understand the role of indigenous genetic factors like TCPTFs during Foc infection in the banana. It has been reported that the different TCPTFs are involved in the regulation of JA and SA biosynthesis in plants [2]. Studies in Arabidopsis and tomato plants have shown increase expression of JA, SA and ethylene biosynthesis can make plants susceptible to the Foc infection. Foc infection was reported to hijack the JA pathway, hence infected plants showed a susceptible response [17]. Here, we anticipate that the higher expression of MaTCP2 (4 fold in root), MaTCP4 (5.02 fold in leaf), MaTCP6 (5.1 fold in root), MaTCP9 (5.71 fold in leaf) and MaTCP 11 (16 fold in leaf) in Foc infected tissues can modulate the SA and JA synthesis in Rasthali plants. 


\section{Expression analysis of MaTCP genes in different vegetative and fruit tissues of banana}

Diverge expression pattern of 27 MaTCPTFs was observed in all banana tissues including stem, leaf, fruit finger, unripe peel, unripe pulp, ripe peel and ripe pulp (Fig. 7). In general, the higher expression of MaTCP was found in the stem, leaf and fruit-finger, whereas other developed tissues such as unripe and ripe fruit tissues (peel and pulp) exhibited lower expression of TFs. MaTCP1, MaTCP2, MaTCP3, MaTCP8, MaTCP13, MaTCP17, MaTCP20 and MaTCP21 were exclusively expressed in the leaf tissue and hinted their prominent role in the regulation of leaf morphology and development. Similarly, in ArabidopsisAtTCP1, AtTCP2, AtTCP3 and AtTCP4 showed higher expression in leaf tissues and played role in leaf development, plant growth and senescence [52]. However, MaTCP7, MaTCP 9, MaTCP 11, MaTCP 14, MaTCP 15, MaTCP 19, MaTCP 22, and MaTCP 24 were preferentially expressed in the stem. The highest expression of all TFs perceived in the stem followed by leaf and fruit finger. It is plausible that the MaTCPTFs are having a promising role in plant growth and development. Moreover, expression analysis of MaTCPTFs in various tissues displayed their paramount role in the development of stem and leaves in banana. The divergent expression patterns of MaTCP suggested the functional discrepancy of these TFs in banana development. Similar type patterns were also reported in tobacco, cotton, Medicago and grapevines plants $[3,37,44,45,52]$.

\section{Conclusion}

Total 27 MaTCPTFs were identified in the banana genome and their characterization indicated their promising role in plant development and Foc stress response in banana cv. Rasthali plants. Cis-acting elements analysis revealed that the TCPTFs encompassed regulation and synthesis of various growth and stress response plant hormones. Higher expression of MaTCP13 and MaTCP15 TFs in vegetative tissues such as stem and leaf has revealed their role in growth and development. Higher expression of the MaTCP2, MaTCP4, MaTCP6, MaTCP9 and MaTCP11 TFs in Foc race 1 treated banana plants showed that they might be involved during the Fusarium infection process. Further, these selected TCP TFs belong to the class II subfamily which is responsible for the cell division inhibition and ageing stimulation in plants. Therefore, we suggest that the functional characterization of selected TCPTFs could widen our understanding of their role in genetic control of Foc infection (MaTCP2, MaTCP4, MaTCP6, MaTCP9, MaTCP11) and developmental regulation (MaTCP13 and MaTCP15) in banana plants.

\section{Declarations}

\section{Acknowledgement:}

The authors would like to thank Biotechnology Industry Research Assistance Council (BIRAC) for banana biofortification project grant (BIRAC/TechTransfer/08/I2/QUT-BBF dated 04.09.2012) and the Department of Biotechnology (DBT), Government of India for the grant (BT/PR25789/GET/119/97/2017) under the 
scheme of Genome Engineering Technology and their Applications. SC is thankful to Panjab University Chandigarh and SK thankful to the Central University of Punjab for PhD registration.

Authors' Contributions: S.T. conceived and designed the research. S.C and SK performed computational and qRT-PCR analysis. S.T., S.C. and T.R.U. analysed the data. S.C and S.T wrote the manuscript.

Conflict of interest: The authors declare that they have no conflict of interest.

Funding: This work was supported by the Department of Biotechnology (DBT), Government of India.

Data Availability: All data have been presented in the manuscript.

Ethics Approval and Consent to Participate: Not applicable.

Consent for publication: Not applicable.

\section{References}

1. Martín-Trillo, M., \& Cubas, P. (2010). TCP genes: a family snapshot ten years later. Trends in Plant Science, 15(1), 31-39. https://doi.org/10.1016/j.tplants.2009.11.003

2. Dhaka, N., Bhardwaj, V., Sharma, M. K., \& Sharma, R. (2017). Evolving tale of TCPs: New paradigms and old lacunae. Frontiers in Plant Science, 8(April), 1-8. https://doi.org/10.3389/fpls.2017.00479

3. Chen, L., Chen, Y. Q., Ding, A. M., Chen, H., Xia, F., Wang, W. F., \& Sun, Y. H. (2016). Genome-wide analysis of TCP family in tobacco. Genetics and Molecular Research, 15(2), 1-14. https://doi.org/10.4238/gmr.15027728

4. Huo, Y., Xiong, W., Su, K., Li, Y., Yang, Y., Fu, C., ... Sun, Z. (2019). Genome-Wide Analysis of the TCP Gene Family in Switchgrass (Panicum virgatum L.). International Journal of Genomics, 2019. https://doi.org/10.1155/2019/8514928

5. Navaud, O., Dabos, P., Carnus, E., Tremousaygue, D., \& Hervé, C. (2007). TCP transcription factors predate the emergence of land plants. Journal of molecular evolution, 65(1), 23-33.

6. Hu, Z., Yamauchi, T., Yang, J., Jikumaru, Y., Tsuchida-Mayama, T., Ichikawa, H., ... Yamaguchi, S. (2014). Strigolactone and cytokinin act antagonistically in regulating rice mesocotyl elongation in darkness. Plant and Cell Physiology, 55(1), 30-41.

7. Lan, J., \& Qin, G. (2020). The regulation of CIN-like TCP transcription factors. International Journal of Molecular Sciences, 21(12), 1-17. https://doi.org/10.3390/ijms21124498

8. Hervé, C., Dabos, P., Bardet, C., Jauneau, A., Auriac, M. C., Ramboer, A., ... Tremousaygue, D. (2009). In vivo interference with AtTCP20 function induces severe plant growth alterations and deregulates the expression of many genes important for development. Plant physiology, 149(3), 1462-1477.

9. Zhou, L., Zhang, J., Yan, J., \& Song, R. (2011). Two transposable element insertions are causative mutations for the major domestication gene teosinte branched 1 in modern maize. Cell research, 21(8), 1267-1270. 
10. Waheed, S., \& Zeng, L. (2020). The Critical Role of miRNAs in Regulation of Flowering Time and Flower Development. Genes, 11(3), 319.

11. Li, S., \& Zachgo, S. (2013). TCP 3 interacts with R2R3-MYB proteins, promotes flavonoid biosynthesis and negatively regulates the auxin response in A rabidopsis thaliana. The Plant Journal, 76(6), 901913.

12. Steiner, E., Efroni, I., Gopalraj, M., Saathoff, K., Tseng, T.-S., Kieffer, M., ... Weiss, D. (2012). The Arabidopsis O-linked $\mathrm{N}$-acetylglucosamine transferase SPINDLY interacts with class I TCPs to facilitate cytokinin responses in leaves and flowers. The Plant Cell, 24(1), 96-108.

13. Davière, J.-M., Wild, M., Regnault, T., Baumberger, N., Eisler, H., Genschik, P., \& Achard, P. (2014). Class I TCP-DELLA interactions in inflorescence shoot apex determine plant height. Current Biology, 24(16), 1923-1928.

14. Wang, X., Gao, J., Zhu, Z., Dong, X., Wang, X., Ren, G., ... Kuai, B. (2015). TCP transcription factors are critical for the coordinated regulation of isochorismate synthase 1 expression in Arabidopsis thaliana. The Plant Journal, 82(1), 151-162.

15. Pozo, M. J., Van Loon, L. C., \& Pieterse, C. M. J. (2004). Jasmonates-signals in plant-microbe interactions. Journal of Plant Growth Regulation, 23(3), 211-222.

16. Ke, Y., Kang, Y., Wu, M., Liu, H., Hui, S., Zhang, Q., ... Wang, S. (2019). Jasmonic acid-involved OsEDS1 signaling in Rice-bacteria interactions. Rice, 12(1), 1-12.

17. Thatcher, L. F., Manners, J. M., \& Kazan, K. (2009). Fusarium oxysporum hijacks COI1-mediated jasmonate signaling to promote disease development in Arabidopsis. The Plant Journal, 58(6), 927939.

18. Di, X., Gomila, J., \& Takken, F. L. W. (2017). Involvement of salicylic acid, ethylene and jasmonic acid signalling pathways in the susceptibility of tomato to Fusarium oxysporum. Molecular Plant Pathology, 18(7), 1024-1035. https://doi.org/10.1111/mpp.12559

19. Nicolas, M., \& Cubas, P. (2016). TCP factors: New kids on the signaling block. Current Opinion in Plant Biology, 33, 33-41. https://doi.org/10.1016/j.pbi.2016.05.006

20. Voora, V., Larrea, C., \& Bermudez, S. (2020). Global Market Report: Bananas. International Institute for Sustainable Development.

21. Maymon, M., Sela, N., Shpatz, U., Galpaz, N., \& Freeman, S. (2020). The origin and current situation of Fusarium oxysporum f. sp. cubense tropical race 4 in Israel and the Middle East. Scientific Reports, 10(1), 1-11. https://doi.org/10.1038/s41598-020-58378-9

22. Altendorf, S. (2019). Banana Fusarium wilt tropical race 4: a mounting threat to global banana markets? Banana Fusarium wilt tropical race 4: a mounting threat to global banana markets?

23. Mohandas, S., Sowmya, H. D., Saxena, A. K., Meenakshi, S., Rani, R. T., \& Mahmood, R. (2013). Transgenic banana cv. Rasthali (AAB, Silk gp) harboring Ace-AMP1 gene imparts enhanced resistance to Fusarium oxysporum f. sp. cubense race 1. Scientia Horticulturae, 164, 392-399.

24. D'hont, A., Denoeud, F., Aury, J.-M., Baurens, F.-C., Carreel, F., Garsmeur, O., ... Rouard, M. (2012). The banana (Musa acuminata) genome and the evolution of monocotyledonous plants. Nature, 
488(7410), 213-217.

25. Finn, R. D., Mistry, J., Tate, J., Coggill, P., \& Heger, A. (2014). Pfam: the protein families database. Nuclei. Acids Re.

26. Gasteiger, E., Gattiker, A., Hoogland, C., Ivanyi, I., Appel, R. D., \& Bairoch, A. (2003). ExPASy: the proteomics server for in-depth protein knowledge and analysis. Nucleic acids research, 31(13), 3784-3788.

27. Larkin, M. A., Blackshields, G., Brown, N. P., Chenna, R., McGettigan, P. A., McWilliam, H., ... Lopez, R. (2007). Clustal W and Clustal X version 2.0. bioinformatics, 23(21), 2947-2948.

28. Kumar, S., Stecher, G., Li, M., Knyaz, C., \& Tamura, K. (2018). MEGA X: molecular evolutionary genetics analysis across computing platforms. Molecular biology and evolution, 35(6), 1547.

29. Krzywinski, M., Schein, J., Birol, I., Connors, J., Gascoyne, R., Horsman, D., ... Marra, M. A. (2009). Circos: an information aesthetic for comparative genomics. Genome research, 19(9), 1639-1645.

30. Hu, B., Jin, J., Guo, A.-Y., Zhang, H., Luo, J., \& Gao, G. (2015). GSDS 2.0: an upgraded gene feature visualization server. Bioinformatics, 31(8), 1296-1297.

31. Bailey, T. L., Boden, M., Buske, F. A., Frith, M., Grant, C. E., Clementi, L., ... Noble, W. S. (2009). MEME SUITE: tools for motif discovery and searching. Nucleic acids research, 37(suppl_2), W202-W208.

32. Du, Z., Zhou, X., Ling, Y., Zhang, Z., \& Su, Z. (2010). agriGO: a GO analysis toolkit for the agricultural community. Nucleic acids research, 38(suppl_2), W64-W70.

33. Ghag, S. B., Shekhawat, U. K. S., \& Ganapathi, T. R. (2014). Transgenic banana plants expressing a Stellaria media defensin gene (Sm-AMP-D1) demonstrate improved resistance to Fusarium oxysporum. Plant Cell, Tissue and Organ Culture (PCTOC), 119(2), 247-255.

34. Sunisha, C., Sowmya, H. D., Usharani, T. R., Umesha, M., Gopalkrishna, H. R., \& Saxena, A. (2020). Deployment of stacked antimicrobial genes in banana for stable tolerance against fusarium oxysporum f. sp. cubense through genetic transformation. Molecular biotechnology, 62(1), 8-17.

35. Kaur, N., Alok, A., Kumar, P., Kaur, N., Awasthi, P., Chaturvedi, S., ... Tiwari, S. (2020). CRISPR/Cas9 directed editing of lycopene epsilon-cyclase modulates metabolic flux for $\beta$-carotene biosynthesis in banana fruit. Metabolic engineering, 59, 76-86.

36. Livak, K. J., \& Schmittgen, T. D. (2001). Analysis of relative gene expression data using real-time quantitative PCR and the 2- $\Delta \Delta C T$ method. methods, 25(4), 402-408.

37. Li, W., Li, D. Di, Han, L. H., Tao, M., Hu, Q. Q., Wu, W. Y., ... Huang, G. Q. (2017). Genome-wide identification and characterization of TCP transcription factor genes in upland cotton (Gossypium hirsutum). Scientific Reports, 7(1), 1-14. https://doi.org/10.1038/s41598-017-10609-2

38. Martín-Rodríguez, J., Leija, A., Formey, D., \& Hernández, G. (2018). The microRNA319d/TCP10 node regulates the common bean - Rhizobia nitrogen-fixing symbiosis. Frontiers in Plant Science, 9(August), 1-13. https://doi.org/10.3389/fpls.2018.01175

39. Ding, S., Cai, Z., Du, H., \& Wang, H. (2019). Genome-wide analysis of TCP family genes in Zea mays I. Identified a role for ZmTCP42 in drought tolerance. International Journal of Molecular Sciences, 
20(11). https://doi.org/10.3390/ijms20112762

40. Song, C. B., Shan, W., Yang, Y. Y., Tan, X. L., Fan, Z. Q., Chen, J. Y., ... Kuang, J. F. (2018).

Heterodimerization of MaTCP proteins modulates the transcription of MaXTH10/11 genes during banana fruit ripening. Biochimica et Biophysica Acta - Gene Regulatory Mechanisms, 1861(7), 613622. https://doi.org/10.1016/j.bbagrm.2018.06.005

41. Moreano, J. P. S., Xu, X., Criollo, C. B. A., Chen, X., Lin, Y., Munir, N., \& Lai, Z. (2021). Genome-Wide Identification and Comprehensive Analyses of TCP Gene Family in Banana (Musa L.). Tropical Plant Biology, 1-23.

42. Mukhopadhyay, P., \& Tyagi, A. K. (2015). OsTCP19 influences developmental and abiotic stress signaling by modulating ABI4-mediated pathways. Scientific Reports, 5, 1-12.

https://doi.org/10.1038/srep09998

43. Jain, M., Khurana, P., Tyagi, A. K., \& Khurana, J. P. (2008). Genome-wide analysis of intronless genes in rice and Arabidopsis. Functional and Integrative Genomics, 8(1), 69-78.

https://doi.org/10.1007/s10142-007-0052-9

44. Zheng, K., Ni, Z., Qu, Y., Cai, Y., Yang, Z., Sun, G., \& Chen, Q. (2018). Genome-wide identification and expression analyses of TCP transcription factor genes in Gossypium barbadense. Scientific Reports, 8(1), 1-16. https://doi.org/10.1038/s41598-018-32626-5

45. Jiu, S., Xu, Y., Wang, J., Wang, L., Wang, S., Ma, C., ... Zhang, C. (2019). Genome-Wide Identification, Characterization, and Transcript Analysis of the TCP Transcription Factors in Vitis vinifera. Frontiers in Genetics, 10, 1-18. https://doi.org/10.3389/fgene.2019.01276

46. Sheshadri, S. A., Nishanth, M. J., \& Simon, B. (2016). Stress-mediated cis-element transcription factor interactions interconnecting primary and specialized metabolism in planta. Frontiers in plant science, 7, 1725 .

47. Baima, S., Possenti, M., Matteucci, A., Wisman, E., Altamura, M. M., Ruberti, I., \& Morelli, G. (2001). The Arabidopsis ATHB-8 HD-zip protein acts as a differentiation-promoting transcription factor of the vascular meristems. Plant physiology, 126(2), 643-655.

48. Li, C., Qiao, Z., Qi, W., Wang, Q., Yuan, Y., Yang, X., ... Songa, R. (2015). Genome-wide characterization of cis-acting DNA targets reveals the transcriptional regulatory framework of Opaque2 in maize. Plant Cell, 27(3), 532-545. https://doi.org/10.1105/tpc.114.134858

49. Dong, H., Li, Y., Fan, H., Zhou, D., \& Li, H. (2019). Quantitative proteomics analysis reveals resistance differences of banana cultivar 'Brazilian'to Fusarium oxysporum f. sp. cubense races 1 and 4 . Journal of proteomics, 203, 103376.

50. Arie, T. (2019). Fusarium diseases of cultivated plants, control, diagnosis, and molecular and genetic studies, 44(4), 275-281. https://doi.org/10.1584/jpestics.J19-03

51. Barboza-barquero, L., Albertazzi, F. J., Chac, R., \& Rivera-m, W. (2020). Physiological and Molecular Plant Pathology Transcription factors controlling biotic stress response in potato plants, 112(March). https://doi.org/10.1016/j.pmpp.2020.101527 
52. Wang, H., Wang, H., Liu, R., Xu, Y., Lu, Z., \& Zhou, C. (2018). Genome-wide identification of TCP family transcription factors in medicago truncatula reveals significant roles of miR319-targeted TCPs in nodule development. Frontiers in Plant Science, 9(June), 1-16.

https://doi.org/10.3389/fpls.2018.00774

\section{Figures}

a) $\quad \begin{aligned} & \text { MaTCP2 } \\ & \text { MaTCP3 } \\ & \text { MaTCP4 } \\ & \text { MaTCP5 } \\ & \text { MaTCP6 } \\ & \text { MaTCP7 } \\ & \text { MaTCP8 } \\ & \text { MaTCP9 } \\ & \text { MaTCP10 } \\ & \text { MaTCP11 } \\ & \text { MaTCP12 } \\ & \text { MaTCP13 } \\ & \text { MaTCP14 } \\ & \text { MaTCP15 } \\ & \text { MaTCP16 } \\ & \text { MaTCP17 } \\ & \text { MaTCP18 } \\ & \text { MaTCP19 } \\ & \text { MaTCP20 } \\ & \text { MaTCP21 } \\ & \text { MaTCP22 } \\ & \text { MaTCP23 } \\ & \text { MaTCP24 } \\ & \text { MaTCP25 } \\ & \text { MaTCP26 } \\ & \text { MaTCP27 } \\ & \text { MaTCP27 }\end{aligned}$
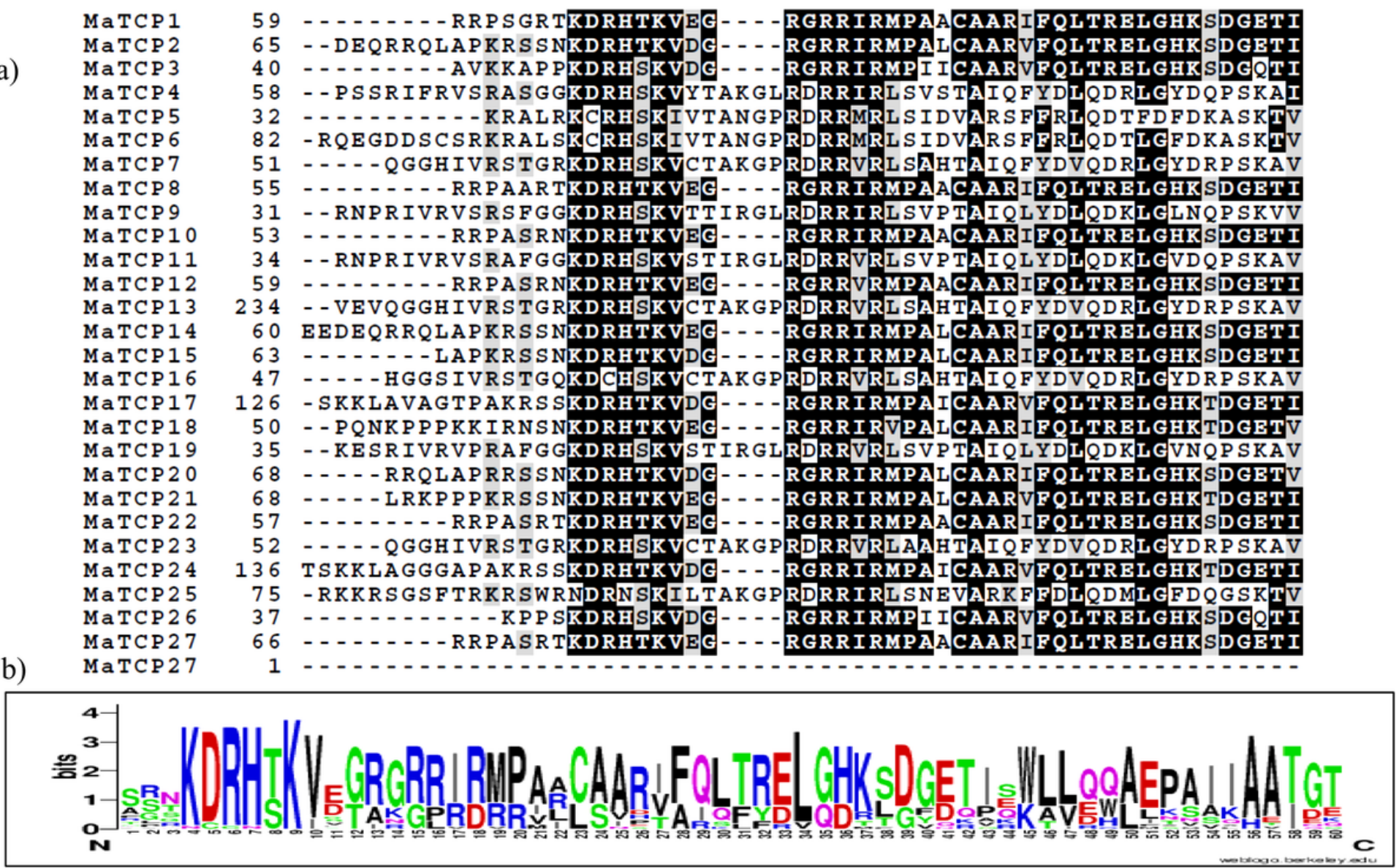

Figure 1

a) Multiple sequence alignment of 27 proteins in the MaTCP family. Each letter represents the one amino acid (aa) and the left column corresponds to the name of genes. The black shaded region represents the highly conserved residue of MaTCP family members. Class I members showed the deletion of four aa in the basic region. b) A sequence logo of TCP domain. The height of the letter represents conservedness of the domain, more the height more the conserved domain. 


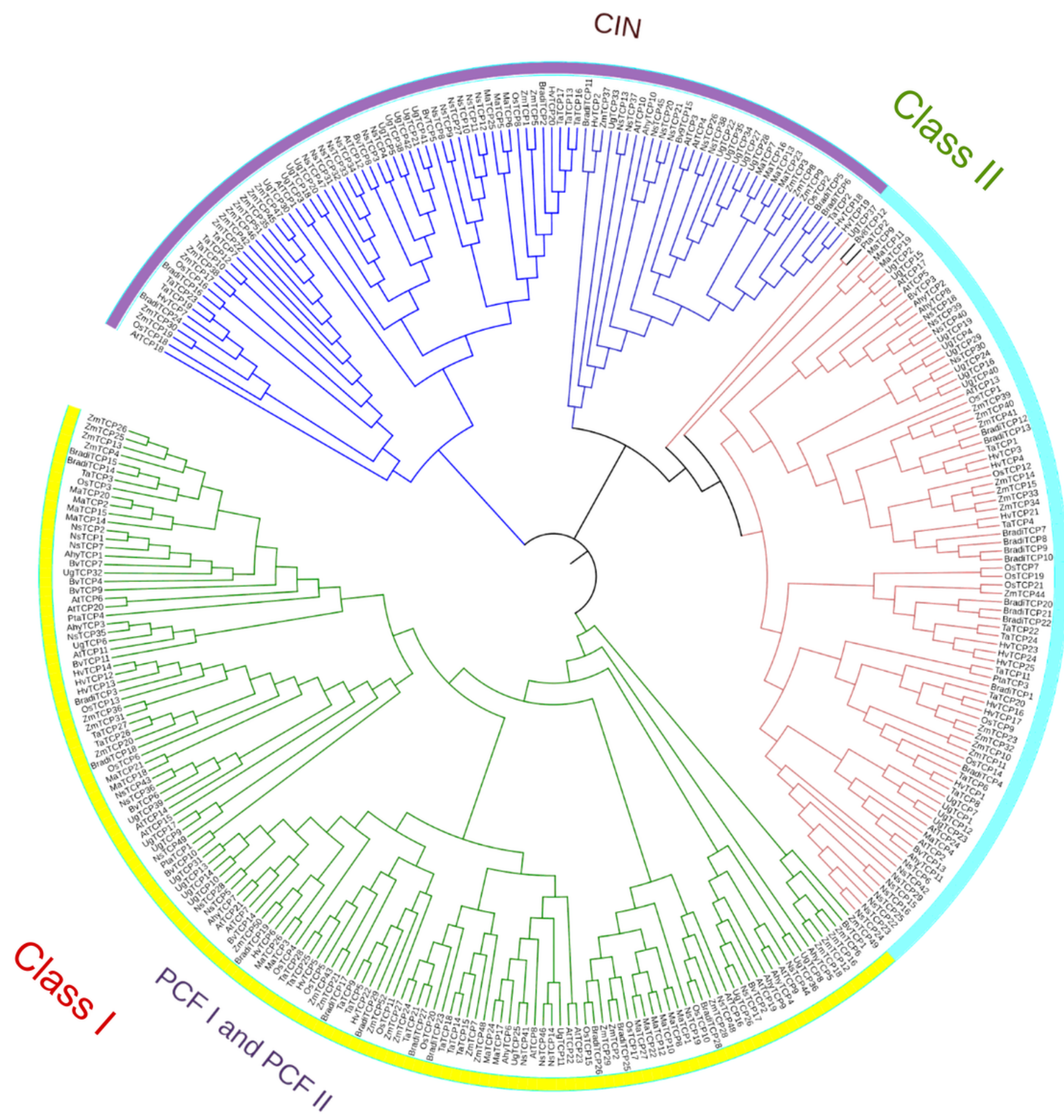

$\frac{\Omega}{2}$

Figure 2

Phylogenetic tree of predicted TCP proteins from Arabidopsis, Arachis, Beta vulgaris, Nicotiana, Utricularia, Brachypodium, Hordeum vulgare, Oryza sativa var. indica, Triticum, Zea mays, Pinus and banana. The phylogenetic tree was drawn by MegaX with the neighbour-joining method using the bootstrap value (1000 replicates) and the Poisson model. 


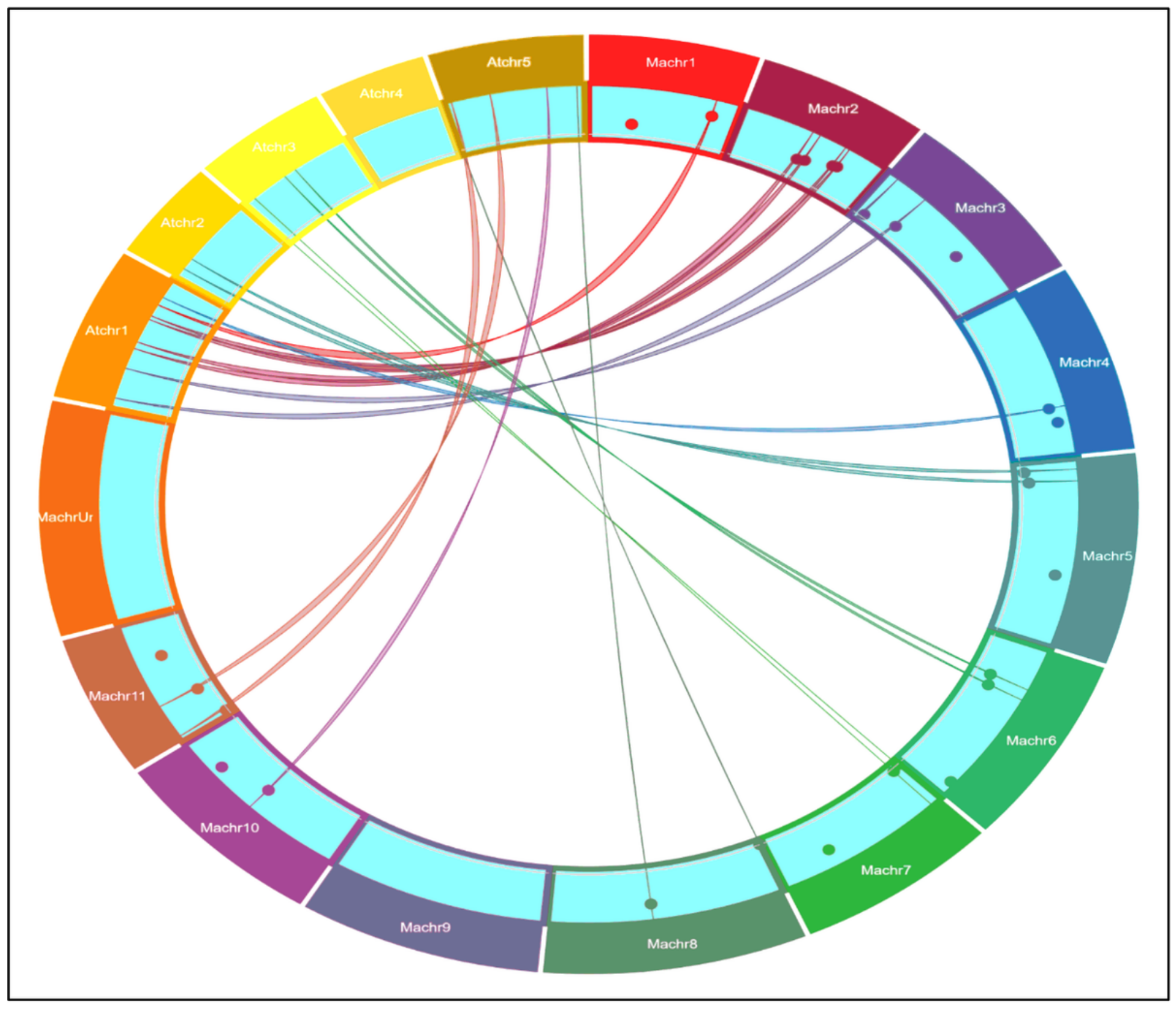

Figure 3

Synteny analysis of Arabidopsis and banana (Musa) MaTCP TFs using the circos plot. The end of the lines represents the orthologous genes from Arabidopsis and Musa. 


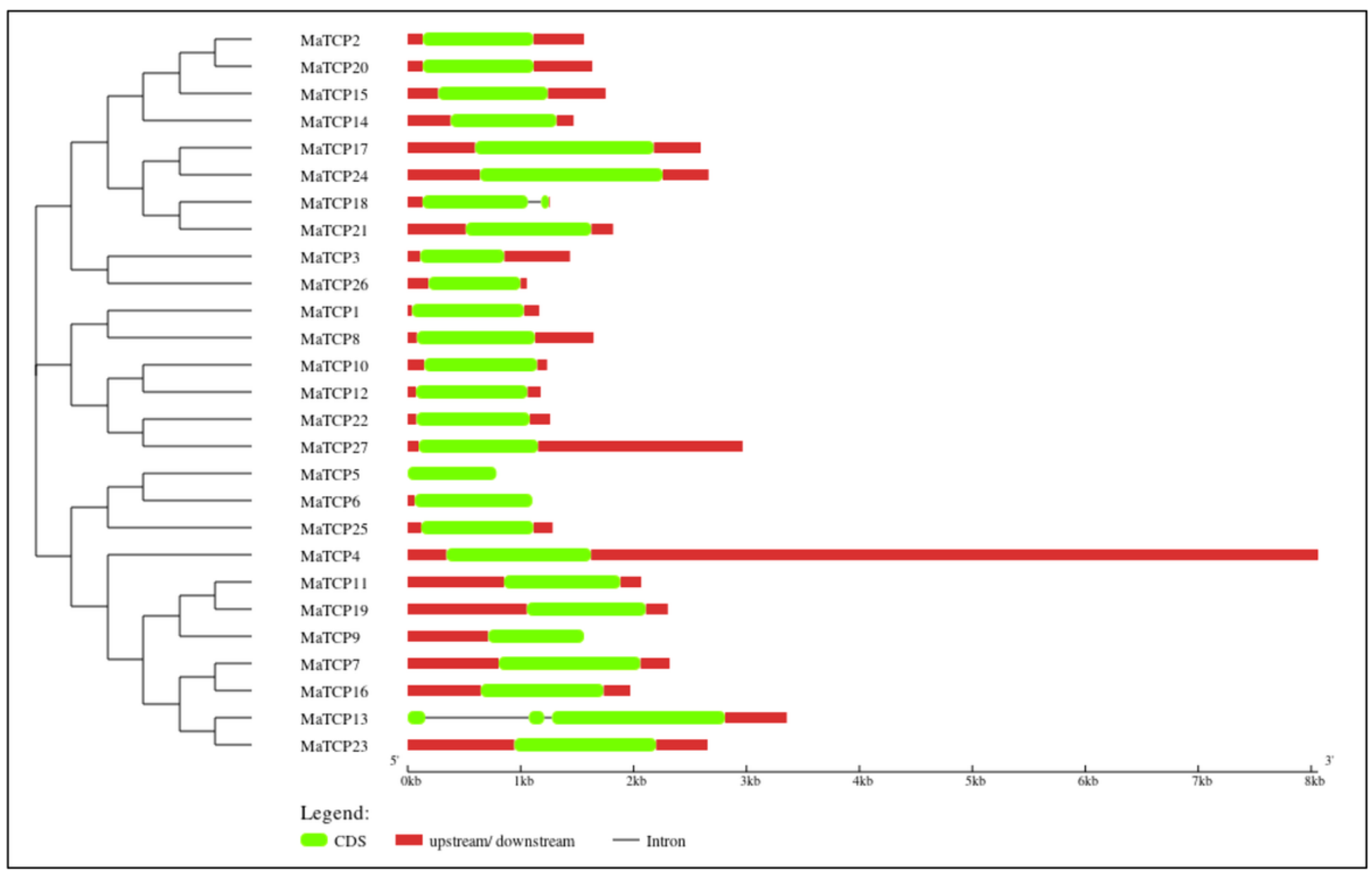

\section{Figure 4}

Exon/intron distribution in the MaTCP TF gene family. The Red line represents the untranslated region and the green lines represent exons. A scale (bottom) to estimate the size of exons and introns is provided. 


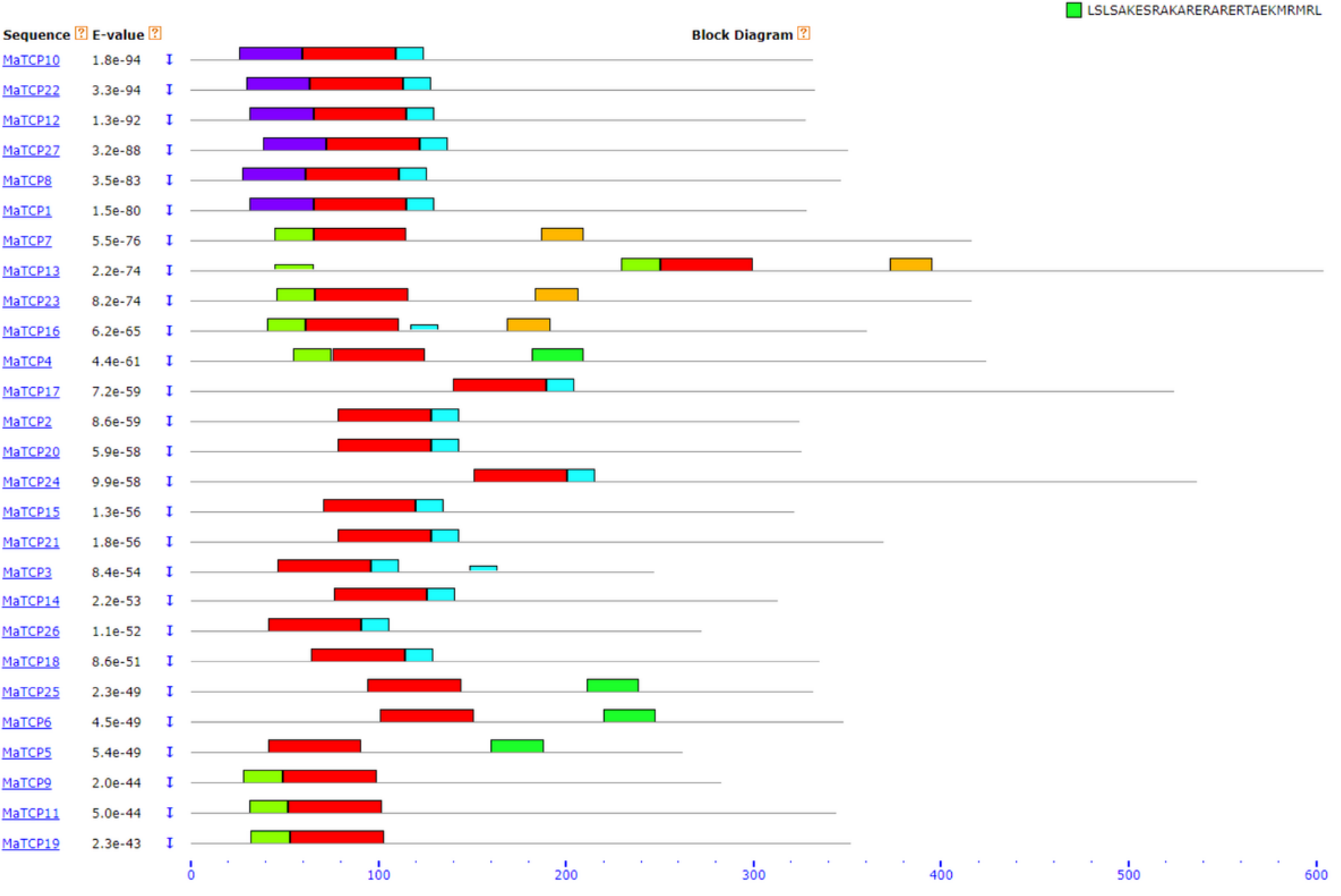

\section{Figure 5}

Base sequence diagram of 27 MaTCP family proteins. Each coloured box represents a motif of the protein and lower scale is used to estimate the size of the protein. 
0.0

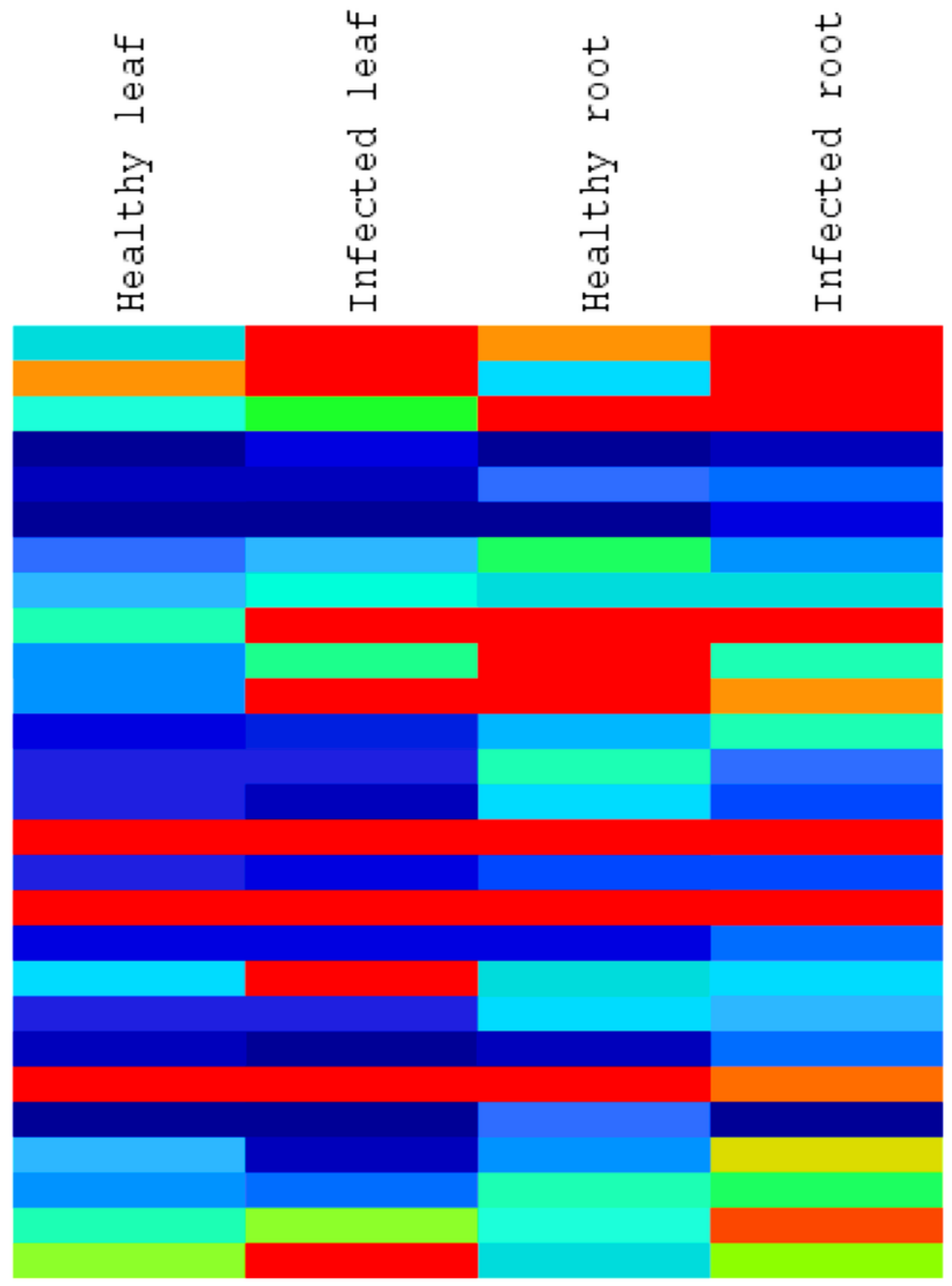

MaTCP1 MaTCP2 MaTCP 3 MaTCP 4 MaTCP 5 MaTCPG MaTCP 7 MaTCP8 MaTCPS MaTCP10 MaTCP 11 MaTCP12 MaTCP13 $\mathrm{MaTCP} 14$ MaTCP 15 MaTCP 16 MaTCP 17 MaTCP18 MaTCP19 MaTCP2D MaTCP21 MaTCP22 MaTCP2 3 MaTCP2 4 MaTCP2 5 MaTCP2 6 MaTCP27

\section{Figure 6}

The heat map display expression profile of MaTCP genes for Foc race 1 infected root and leaf tissues in comparison with healthy leaf and root of banana. The blue color in color range constitutes lower expression, green exhibits moderate while red color is for higher expression. 


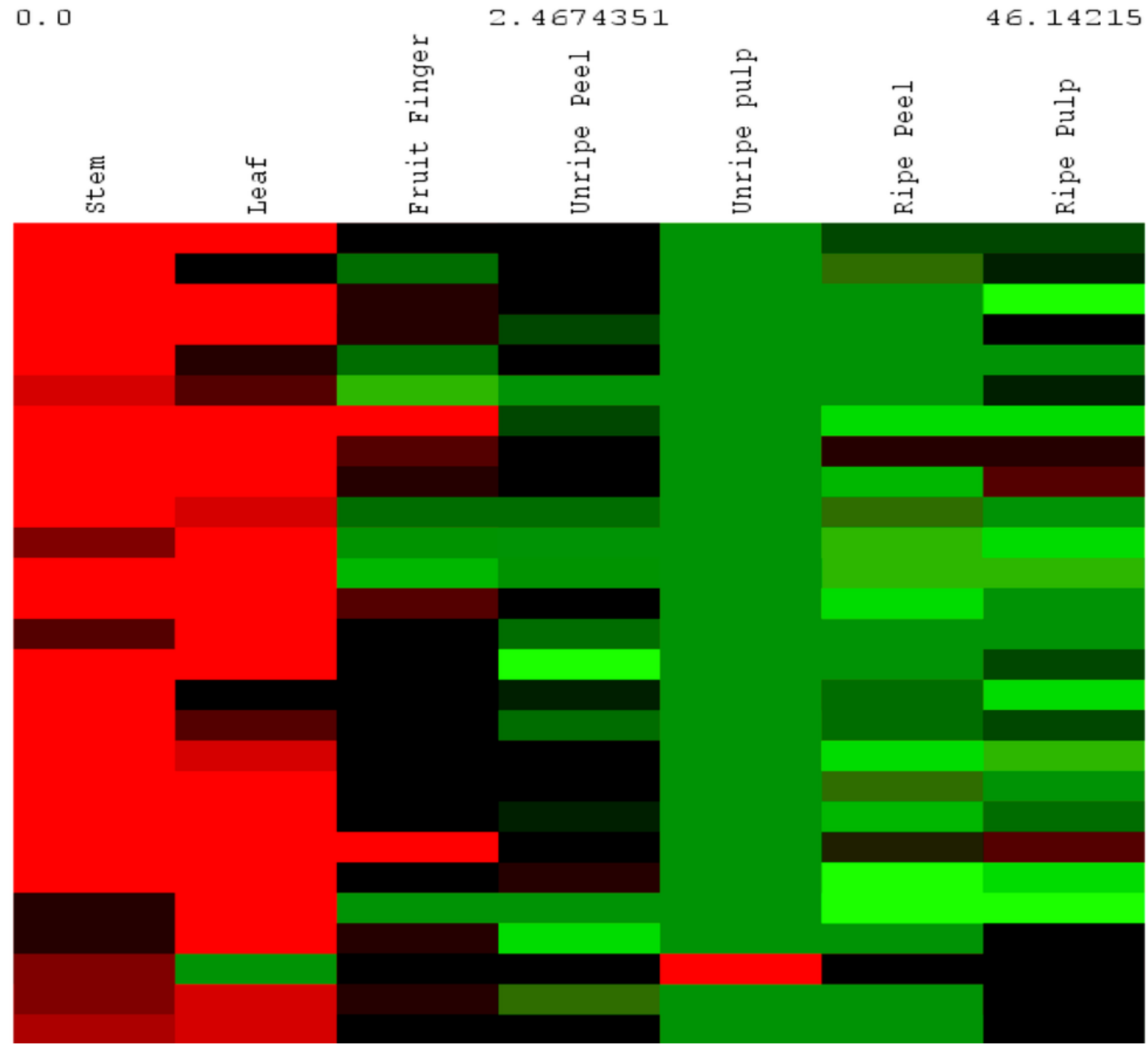

MaTCP 1 MaTCP 2 MaTCP 3 MaTCP 4 MaTCP 5 MaTCP 6 MaTCP 7 MaTCP 8 MaTCP 9 MaTCP 10 MaTCP 11 MaTCP 12 MaTCP 13 MaTCP 14 MaTCP 15 MaTCP 16 MaTCP 17 MaTCP 18 MaTCP 19 MaTCP 20 MaTCP 21 MaTCP 22 MaTCP 23 MaTCP 24 MaTCP 25 MaTCP 26 MaTCP 27

\section{Figure 7}

Heat map depicts relative gene expression pattern of MaTCP genes over different developmental tissues. The color bar represents relative expression value as shown at the upper side of the image. Where red color describes higher expression, green characterize lower expression while black referes to the medium expression.

\section{Supplementary Files}

This is a list of supplementary files associated with this preprint. Click to download.

- S1Table.docx

- S2Table.docx

- S3Table.docx

- S1.tiff 
- S2.tif

- S3.tif

- S4.tif

- S5.tif

Page $21 / 21$ 